\title{
Control of Suddenly Expanded Flows from Correctly Expanded and under Expanded Nozzlesat Supersonic Mach number for Area Ratio 2.56
}

\author{
Musavir Bashir ${ }^{1}$, M. Ahmed Ali Baig ${ }^{2}$, Ahmad Faris Ismail ${ }^{3}$, and S. A. Khan ${ }^{4}$ \\ ${ }^{1}$ Research Scholar, School of Aerospace Engineering, USM, Penang, Malaysia \\ ${ }^{2}$ Professor, Department of Mechanical Engineering, CMR Technical Campus, Hyderabad, India \\ ${ }^{3,4}$ Professor, Department of Mechanical Engineering, Faculty of Engineering, IIUM, Malaysia
}

\begin{abstract}
Airflow from convergent-divergent axisymmetric nozzles expanded into circular duct of higher area were studied experimentally, with the main focus on the flow development in the duct. The flow parameters considered in this investigation are the Mach number at the nozzle exit and the level of expansion. The geometrical parameters considered are the area ratio and the L/D ratio of the duct. To control on the flow field developed in the duct, four tiny jets of $0.5 \mathrm{~mm}$ radius located at $90^{\circ}$ intervals at $6.5 \mathrm{~mm}$ radius from the centre of the nozzle exit were employed as the flow regulators. The Mach number in this case was 1.48. The area ratio of the study was 2.56. The nozzle pressure ratio (NPR) used was from 3.27 and 5.35 respectively which corresponds to ideally expanded and under expanded cases. The $L / D$ ratio of the enlarged duct was varied from 10 to 1 . From the results it is found that there is a jump in the wall pressure when flow passes through first oblique shock and this trend is repeated for all the cases.From the results it is found that the entire flow field is full of waves and flow remains identical with and without control when the jets were operated under favourable and adverse pressure gradient, it is also observed that when flow regulators in the form of tiny jets are employed they do not disturb the flow of duct.
\end{abstract}

\section{Introduction}

Flow field of abrupt axi-symmetric expansion is a complex phenomenon which results in the flow separation, flow recirculation and re-attachment. A shear layer into two main regions may divide such a flow field, one is the flow recirculation region and the other the main flow region. The point at which the dividing streamline strikes the wall is called the re-attachment point.Nusselt is the one who conducted tests with gas flow through ducts with sudden enlargement. The effect of boundary layer on sonic flow through an abrupt crosssectional area was studied experimentally by Wick [1]. He found that the pressure in the corner of was related to the boundary layer type and thickness before the expansion. The base corner was assumed to be a sump with two supplies of mass. The first was the boundary layer flow around the corner and the second source was the back flow in the boundary layer along the wall. This back flow occurred because of the pressure difference across the shock wave originating where the jet meets the wall.

Badrinarayanan[3] investigated experimentally the base flows at supersonic speeds for flow at 2-D and 3-D bodies with blunt base were made at $\mathrm{M}=2$. Ackeret[4]studied special features of internal flow. He concluded that there is a pre-dominant role played by the equation of continuity, especially if compressibility is involved and in aeronautics big deflection of the air streams are avoided as far as possible but in ducted flow, they may be quite common. If the width of the duct is not growing too fast along its length, separation is followed by re-attachment. He observed that, in case of internal flow also, three-dimensional boundary layers can appear as in external flow. He presented the article on the aspects of internal flow covering different types of internal flows describing some of the aspects of separation, re-attachment and pressure fluctuations that are associated with sudden enlargement flows.

Rathakrishnan and Sreekanth[5] conducted tests for flows from convergent nozzles up to sonic Mach numbers. In their experiments, the flow of air from a plenum chamber to a circular cross-section constant area tube was made to expand suddenly by having an abrupt change in cross-sectional area. The pressure ratios covered ranged from 1.1 to 3.0 and the area ratio used were 2.78 to 8.38 . For an optimum performance of flow through pipes with sudden enlargement, it is not sufficient if the base pressure minimization alone is considered. The total pressure loss must also be taken into account.

Selby [6] studied the 3-D separated flow associated with swept rearward facing steps. Results have indicated that geometric modifications in the region downstream of the step where the span-wise vortex is formed has little effect on the extent of the separated flow, while "conical-lip" and "vortex-trough" base 
modifications lead to significant reduction in re-attachment distances. The "conical-lip" modification involves a step lip with variable radius and the "vortex-trough" is grooves in the surface upstream of the step which produces longitudinal vortices.

Rathakrishnan and Ramanaraju[7] investigated the effect of passive control in the form of cavity. The secondary circulation which were induced by the cavities results in the reduction in the flow oscillations and duct $\mathrm{L} / \mathrm{D}=6$ is of significant effect in managing the base pressure. Viswanath and Patil[8] used passive method to control the base flows at Mach 2. They tested model with cavities at base area and also cavities with ventilation. Their tests showed that the models having ventilated cavities with ventilation gave huge reduction in the drag.

Khan and Rathakrishnan[9-15] conducted tests for nozzles with over expansion level of 0.277 . The area ratios covered were 2.56, 3.24, 4.84, and 6.25. The Mach number of their studies was 1.25, 1.3, 1.48, 1.6, 1.8, 2.0, 2.5, and 3.0. They concluded that the pressure in the corner takes lowest value for lowest area ratio at $\mathrm{L} / \mathrm{D}=$ 6 at all the inertia levels tested. The tiny-jets seems is able to modulate the pressure in the base region at Mach 3.0 for lowest area ratio, resulting in 83 per cent decrease in the pressure the base corner.

Khan and Rathakrishnan[10, 13] conducted experiments for area ratio 3.24 and for three supersonic Mach numbers. From their tests theyachieved 95 per cent enhancementin the base pressure. The experimental study to assess the effects of micro jets under the influence of various level of expansion to control the base drag was studied by Khan and others [15-22]. The result was very effective in terms of percentage, as micro jets reduced the base drag without affecting the wall pressure distribution. It is found that many techniques can be used to reduce or even suppress the flow separation.

The above discussion indicates that there is a need to study the flow development in the duct whenever active or passive controls are used to manipulate the flow.

\section{Results and Discussion}

One of the major difficulty encountered while working in the area of sudden expansion is that the flow field in the duct becomes oscillatory because of the "Ejector Pump" action at the base region i.e. the vortices are getting formed at the base because of expansion of the shear layer from the nozzle and getting ejected to the main flow continuously. In the literature this action was known as the “Jet Pump action". This action renders the flow in the duct to become oscillatory. Therefore, it is mandatory on the part of a researcher working in this area to monitor the flow development in the duct. In other words when we employ a control to modify the base pressure level, there is a possibility that the control might augment the flow field in the duct. To take care for this undesirable effect the wall pressure distribution in the duct was measured for all combination of parameters of the present investigation.

\section{Results for Correctly Expanded Jets:}

Wall pressure results for L/D $=10$ to 5 for correctly expanded jets are shown in Figs. 1((a) to (d)). From figure 1(a) it is seen that at first wall pressure tap there is sudden increase in the pressure and this happens twice at $\mathrm{x} / \mathrm{L}=0$ and 0.2 and then flow becomes smooth with marginal changes in the magnitude, When the control was employed it results in the similar behaviour till $\mathrm{x} / \mathrm{L}=0.1$ and in the downstream micro jets are able to break the vortex and hence resultsin lower values of wall pressure, there are some shocks and later the recovery of the flow is smooth becoming almost equal to the back pressure. It was also observed during the tests that when micro jets are activated the noise produced by the jets were quiet and the noise produced are reduced considerably.

Fig. 1 (b) presents the wall pressure distribution for $\mathrm{L} / \mathrm{D}=8$. It is observed that the flow behaviour is similar to that for previous case except the strength of shock and the waviness in the flow due the reflection and recombination of the shock waves.Due to the reduced duct length the wall pressure flow field will be influenced by the back pressure.

Results for L/D = 6 and 5 are shown in Fig. 1((c) to (d)). It is found that with the decrease in the duct length from $\mathrm{L} / \mathrm{D}=10$ and 8 to 6 and 5, there is considerable decrease in the strength of the shock wave resulting in lower values of the wall pressure, and this may be due to the influence of ambient pressure at lower L/Ds. At $\mathrm{L} / \mathrm{D}=10$ as well as at 8 , this large length of the enlarged duct is not influenced by the back pressure resulting in powerful shock wave making the wall pressure for initial few taps becoming almost equal to the atmospheric pressure. The flow field with and without control remains the same. Similar results are seen for L/D = 5.

Results of wall pressure for $\mathrm{L} / \mathrm{D}=4,3,2$ and 1 are shown in Figs. 2((a) to (d)). At $\mathrm{L} / \mathrm{D}=4$ it is seen that the strength of the shock wave is more and hence this leads in the formation of the two strong oblique shocks and remaining are weak shock waves. Here, once again the flow field is identical with and without control and in all four cases and the recovery of the wall pressure is very smooth, and fluctuations in wall pressure field are observed due to the presence of the shock wave. Also, for both the cases the location of reattachment point seems to be at $\mathrm{x} / \mathrm{L}=0.15$. It is also observed that the initial value of the wall pressure 
remains the same for all the L/Ds in the case of correctly expanded jets and this value remains around 0.2 for all the cases of the present study. It is also observed that the flow is attached with the enlarged duct even for L/D $=1$.

\section{Results for Under Expanded Jets:}

Wall pressure results for $\mathrm{L} / \mathrm{D}=10$ to 5 for under expanded jets are shown in Figs. 3((a) to (d)). From figure 3(a) it is seen that there are three strong and six weak oblique shocks which results in compressing the flow and which finally results in increase of the wall pressure in the duct, it is also seen that similar number of expansion waves are present there resulting in the expansion of flow leading to a low values of the wall pressure and this phenomena continue till the end of the pipe length and this was expected as the flow is under expanded and the will continue to expand till it attains the value of the ambient atmospheric pressure. However, when we observe the flow for $\mathrm{L} / \mathrm{D}=8$ as shown in Fig. 3(b) in this case there are four strong oblique shocks and the same number of expansion and this activity is limited to $40 \%$ length of the duct and further in the downstream even though the flow still undergoes expansion and compression of moderate intensity to enable the pressure in the duct is equal to the back pressure value. This strange phenomena for $\mathrm{L} / \mathrm{D}=8$ seems to be due to the reduction in the duct length, and the back pressure will influence the flow in the duct the waves which were spread all along the length of the duct in case of $\mathrm{L} / \mathrm{D}=10$ have been restricted to smaller length of the duct and this value is around $40 \%$ of the total duct length.

Similar results are seen in Fig. 3(c) to (d)) with the exception that in view of the shorter duct lengths namely $\mathrm{L} / \mathrm{D}=6$ and 5 , the flow field in the duct once again is spread over all along the length of the duct. In both the cases we observe six oblique shocks of variable strength and same number of the expansion waves and the very first oblique shock has got the same strength.

When we analyse the results for $\mathrm{L} / \mathrm{D}=4$ and 3 are shown in figure 4 ((a) to (b)), they show similar trends as that of higher L/D ratio with the exception that there are five and four oblique shocks and expansion waves are four and three in numbers, also it is seen that the flow with and without control is the same.

Finally, the results for L/D = 2 and 1 are shown in Fig. 4((c) to (d)), figure shows that the flow remains attached with the duct even at $\mathrm{L} / \mathrm{D}=1$, which implies that in the present tests the level of expansion, inertia level, and geometric parameters are such that the flow is attached and is able to create the suction in base area and this base suction remained constant for all the L/Ds tested. Hence, we may conclude that the entire flow field is full of waves and flow remains identical with and without control when the jets were operated for favourable and adverse pressure gradient, it is also observed that when the regulation in the form of tiny jets are used they do not alter the flow in the duct.

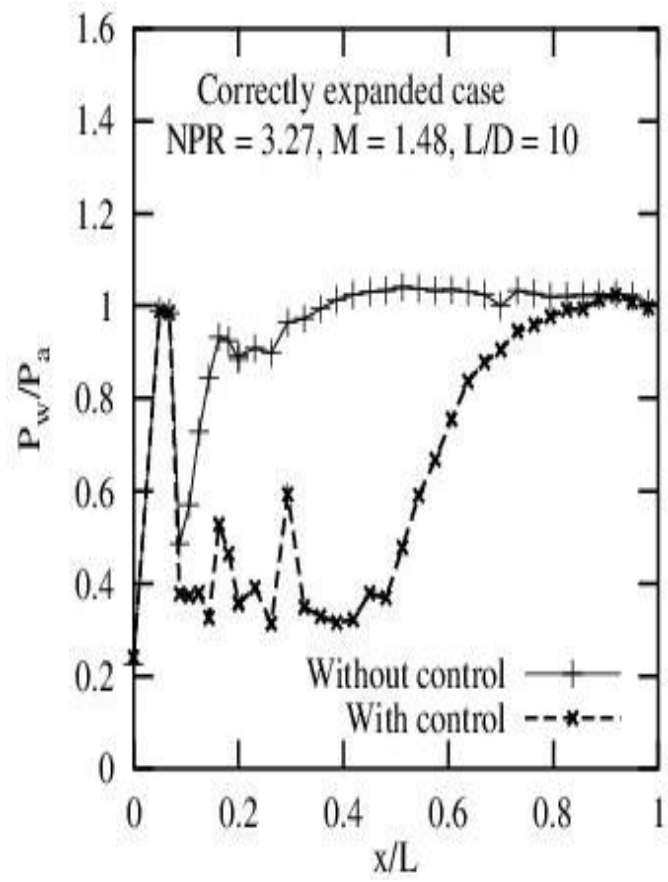

Fig. 1(a)

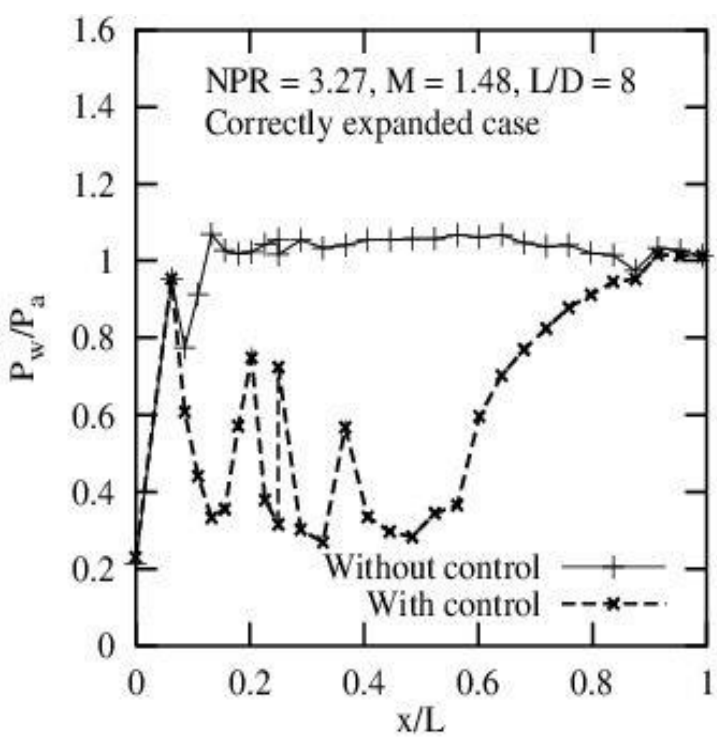

Fig. 1(b) 


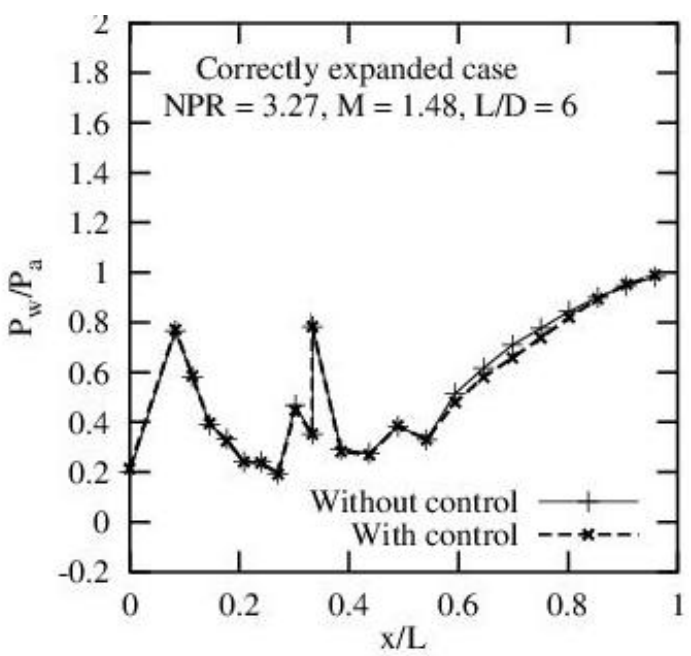

Fig. 1(c)

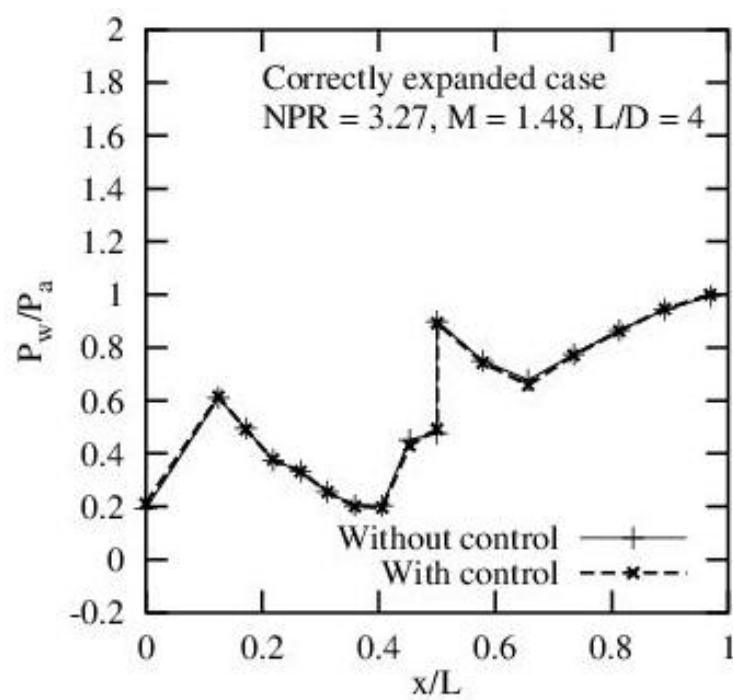

Fig. 2(a)

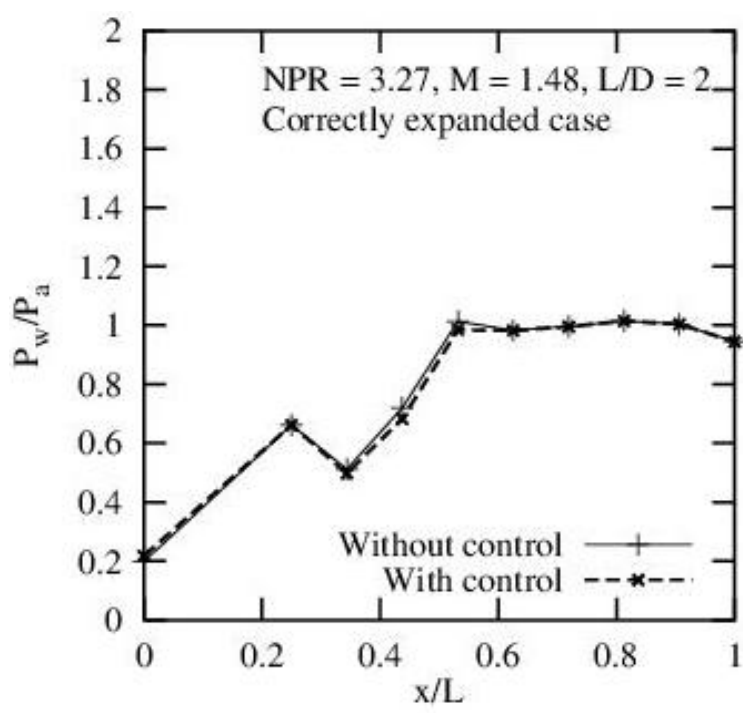

Fig. 2(c)

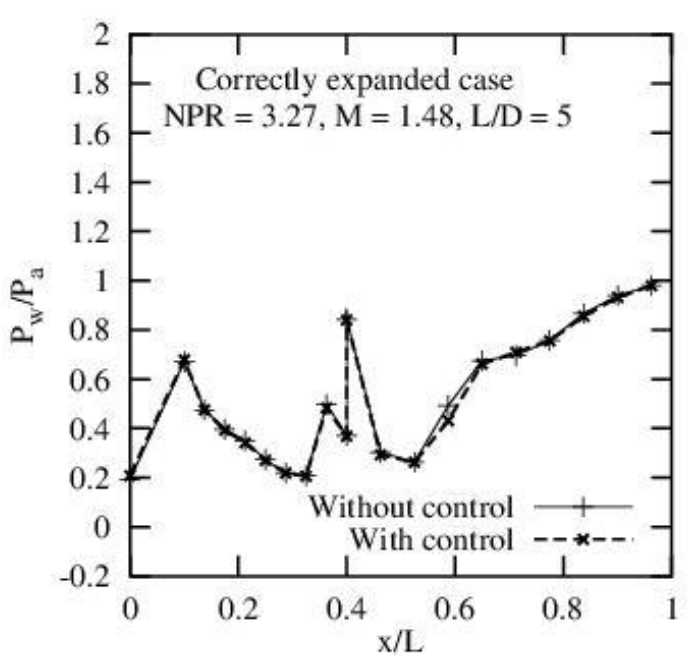

Fig. 1(d)

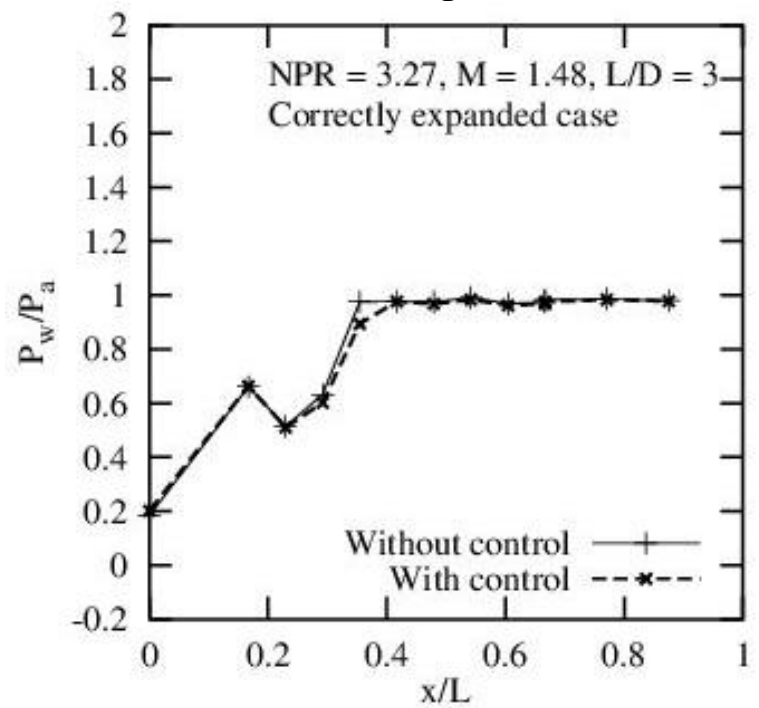

Fig. 2(b)

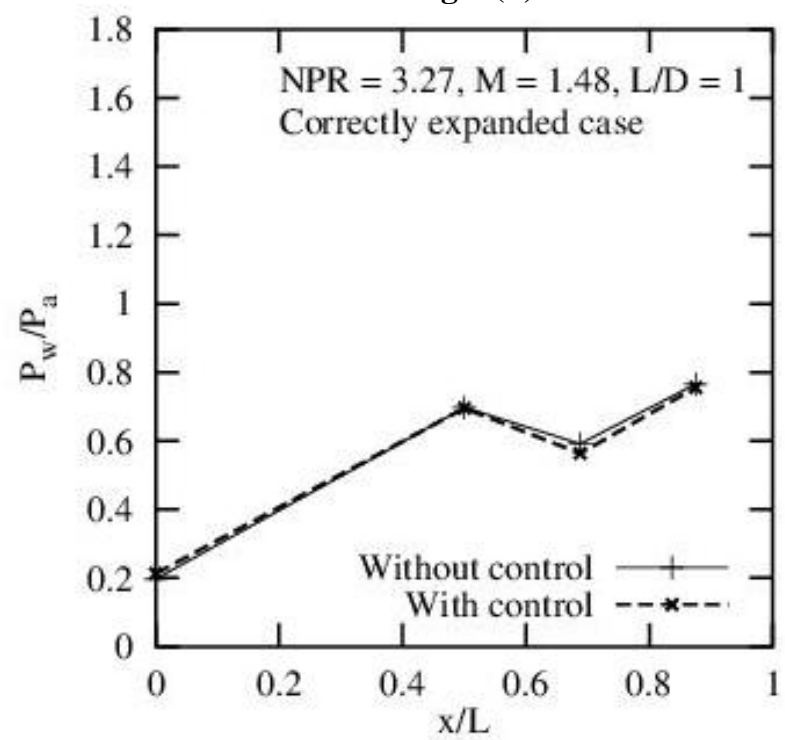

Fig. 2(d) 


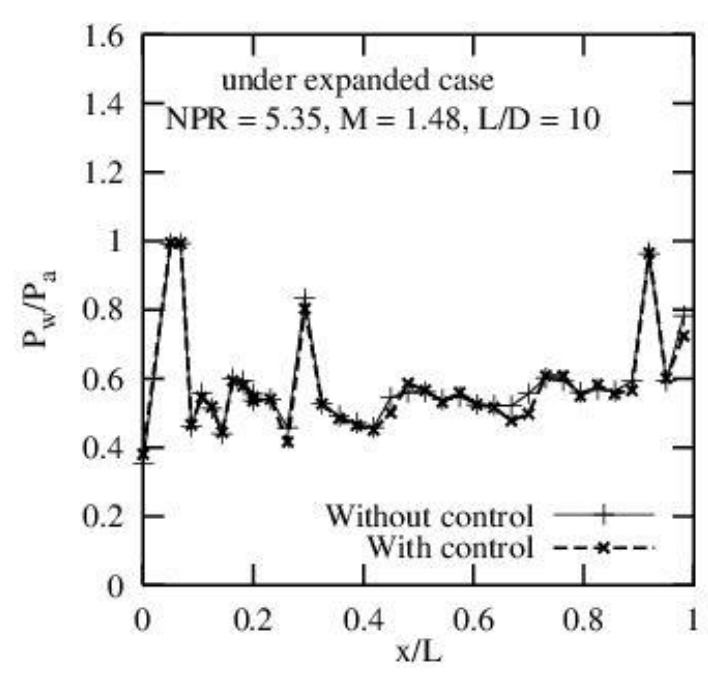

Fig. 3(a)

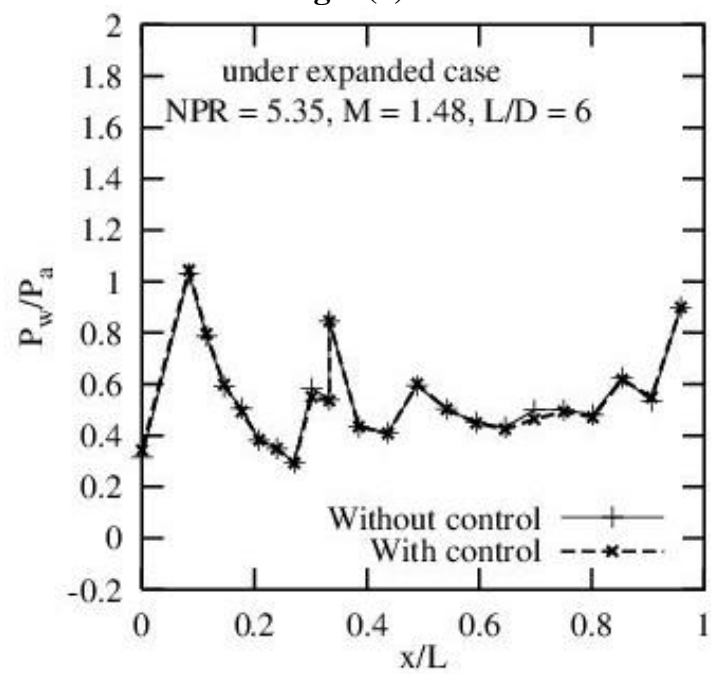

Fig. 3(c)

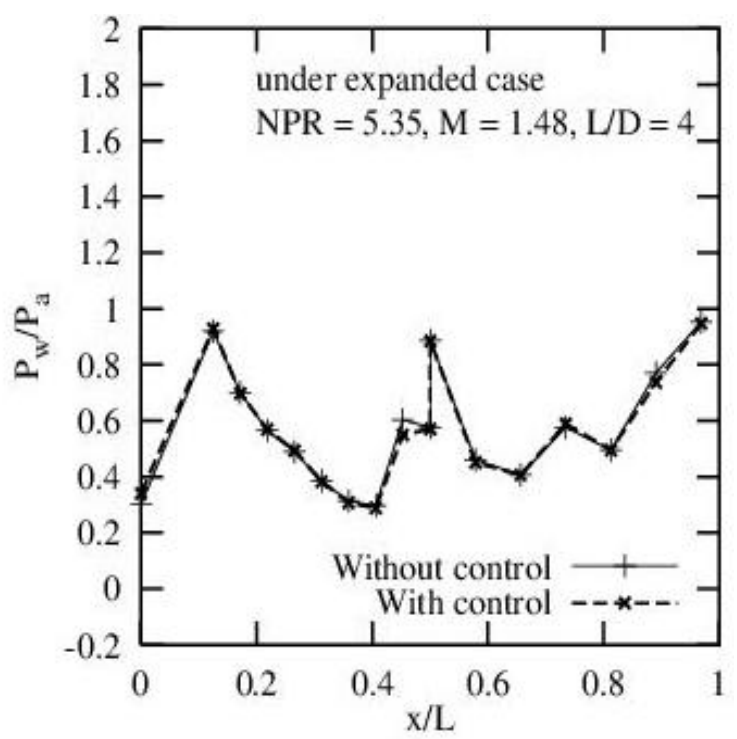

Fig. 4(a)

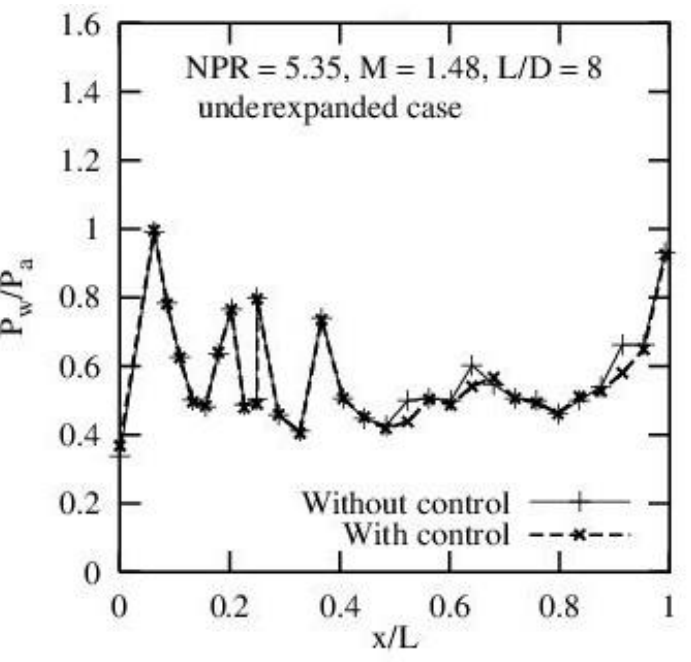

Fig. 3(b)

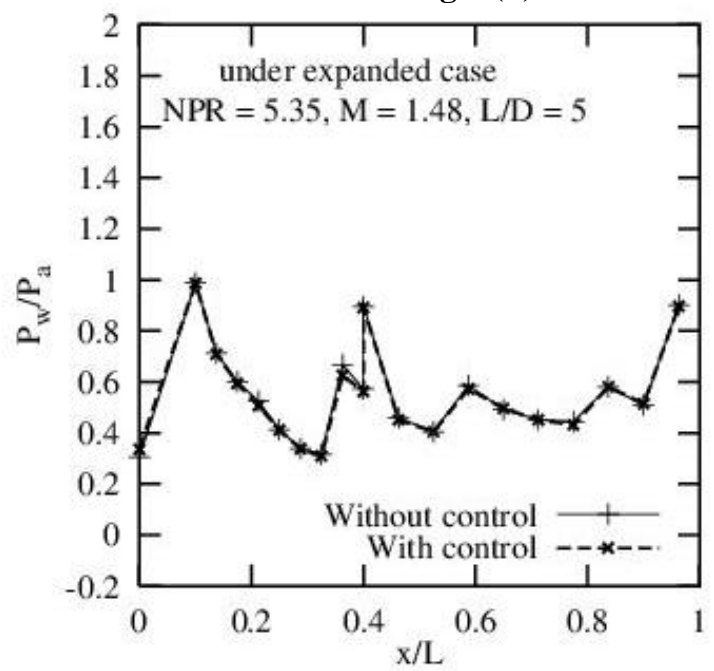

Fig. 3(d)

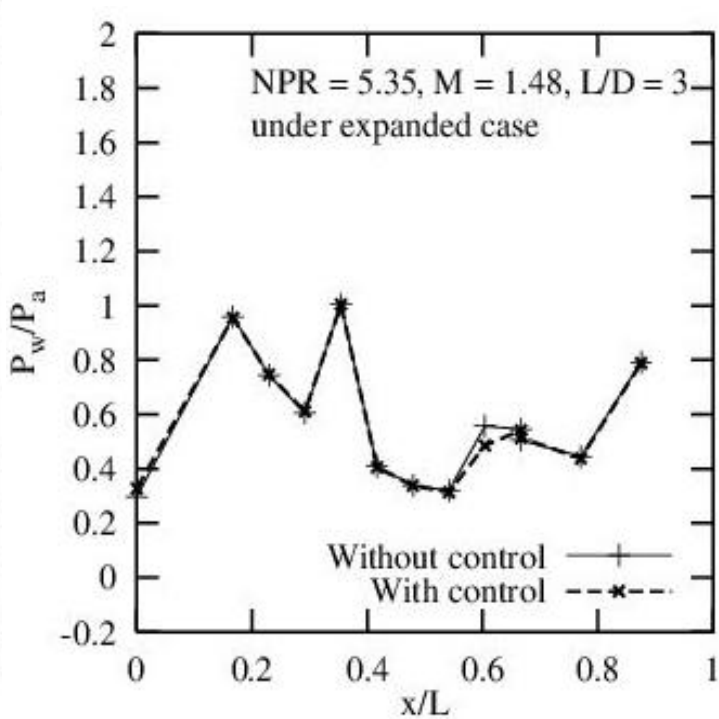

Fig. 4(b) 


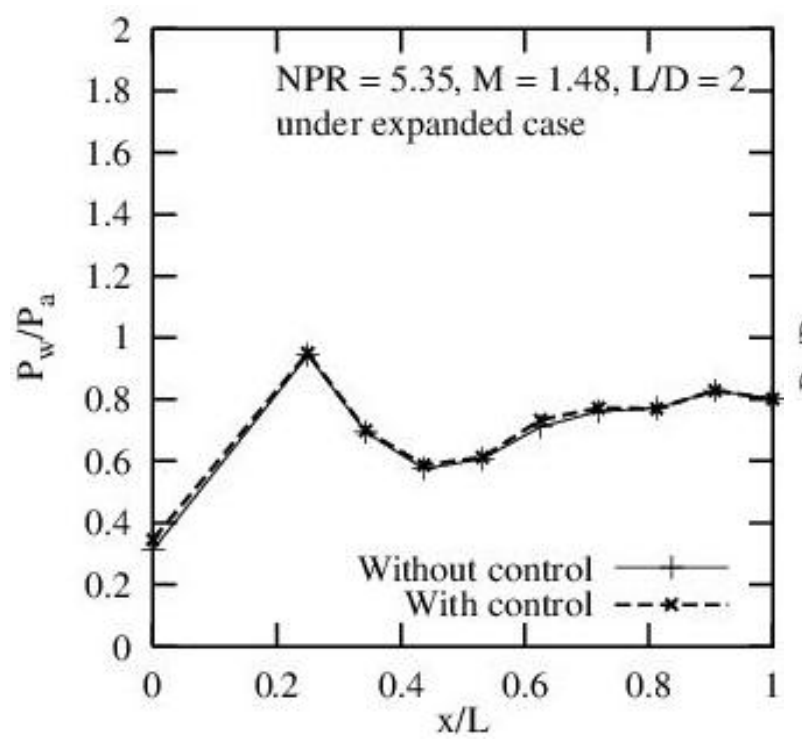

Fig. 4(c)

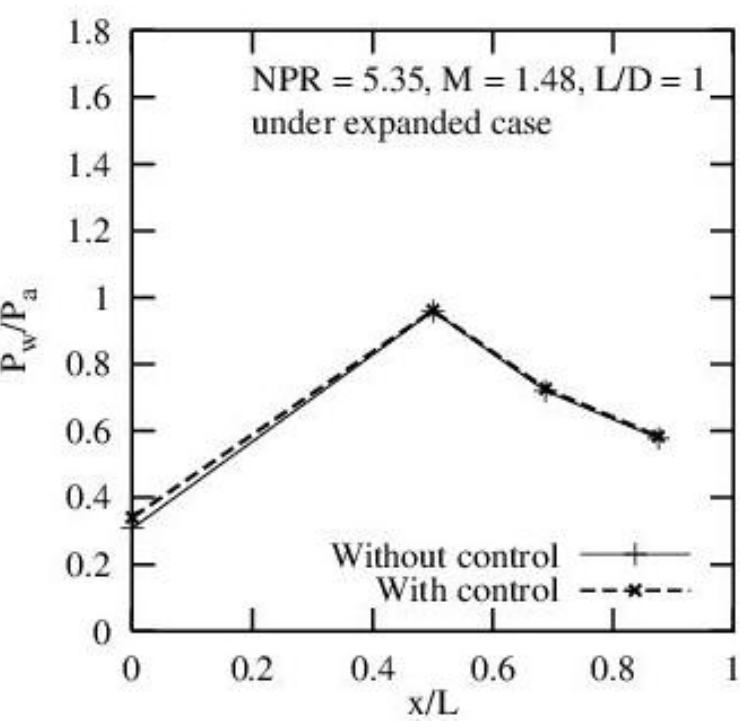

Fig. 4(d)

\section{Conclusion}

From the results and discussions of the present experimental investigation presented in the previous section, the following conclusions can be drawn. When the jets area correctly expanded it is observed that in case of $\mathrm{L} / \mathrm{D}=10$ and 8 , when the control was activated the sound produced by the supersonic jets reduced considerably and the jets were almost quiet. This has given an opening to do the research and control the jet noise with the micro jets. At $\mathrm{x} / \mathrm{L}=0$ the wall pressure value remained constant for under expanded jets as well as correctly expanded jets. Hence, we may conclude that the entire flow field is full of waves and flow remains identical with and without control when the jets were operated in the presence of favourable and adverse pressure gradient, it is also observed that when the active flow regulators were used they do not disturb the wall pressure in the duct. However, the magnitude of the wall pressure remained same for both the L/Ds after the first oblique shock. For lower L/Ds it has reduced in the range from $20 \%$ to $40 \%$, due to the back pressure influence.

\section{References}

[1]. Wick, R.S., The effect of boundary layer on sonic flow through an abrupt cross-sectional area change. Journal of the Aeronautical Sciences, 2012.

[2]. Hoerner, S.F., Base drag and thick trailing edges. Journal of the Aeronautical Sciences, 2012.

[3]. Badrinarayanan, M., An experimental investigation of base flows at supersonic speeds. The Aeronautical Journal, 1961. 65(607): p. 475-482.

[4]. Ackeret, J., Aspects of internal flow. Fluid Mechanics of Internal Flow, 1967: p. 1-24.

[5]. Rathakrishnan, E. and A. Sreekanth. Flows in pipes with sudden enlargement. in 14th Space Technology and Science Symposium. 1984

[6]. Selby, G., Passive control of three-dimensional separated vortical flow associated with swept rearward-facing steps. Journal of Fluids Engineering, 1989. 111(1): p. 99-101.

[7]. Rathakrishnan, E., O. Ramanaraju, and K. Padmanaban, Influence of cavities on suddenly expanded flow field. Mechanics research communications, 1989. 16(3): p. 139-146.

[8]. Viswanath, P., Flow management techniques for base and afterbody drag reduction. Progress in Aerospace Sciences, 1996. 32(2): p. 79-129.

[9]. Khan, S.A. and E. Rathakrishnan, Active control of suddenly expanded flows from overexpanded nozzles. International Journal of Turbo and Jet Engines, 2002. 19(1-2): p. 119-126.

[10]. Khan, S.A. and E. Rathakrishnan, Active Control of Suddenly Expanded Flows from Underexpanded Nozzles. International Journal of Turbo and Jet Engines, 2004. 21(4): p. 233-254.

[11]. Khan, S.A. and E. Rathakrishnan, Control of Suddenly Expanded Flows from Correctly Expanded Nozzles. International Journal of Turbo and Jet Engines, 2004. 21(4): p. 255-278.

[12]. Khan, S. and E. Rathakrishnan, Control of suddenly expanded flow. Aircraft Engineering and Aerospace Technology, 2006. 78(4): p. 293-309.

[13]. Khan, S.A. and E. Rathakrishnan, Control of suddenly expanded flows with micro-jets. International Journal of Turbo and Jet Engines, 2003. 20(1): p. 63-82. 
[14]. Ashfaq, S., S. Khan, and E. Rathakrishnan, Control of Base Pressure with Micro Jets for Area Ratio 2.4. International Review of Mechanical Engineering (IREME), 2014. 8(1): p. 1-10.

[15]. M. Ahmed Ali Baig, S.A. Khan, and E. Rathakrishnan, Active Control Of Base Pressure In Suddenly Expanded Flow For Area Ratio 4.84. International Journal of Engineering Science and Technology, 2012. 4(5): p. 1892-1902.

[16]. Khan, S., M. Bashir, and M.A. Ullah, An investigation of base flow control by wall pressure analysis in a suddenly expansion nozzle. 2016

[17]. Chaudhary, Z., et al., Experimental investigation of the base flow from the nozzles with sudden expansion. International Journal of Applied Engineering Research. 10(92): p. 2015.

[18]. Chaudhary, Z.I., et al., Experimental Investigation on the Effectiveness of Active Control Mechanism on Base Pressure at Low Supersonic Mach Numbers, in Innovative Design and Development Practices in Aerospace and Automotive Engineering2017, Springer. p. 197-209.

[19]. M. Ahmed Ali Baig, et al., Control of base flows with micro jets. International Journal of Turbo and Jet Engines, 2011. 28(1): p. 59-69.

[20]. M. Ahmed Ali Baig, S. A. Khan and E. Rathakrishnan "Effect of Mach number In a Suddenly Expanded Flow for Area Ratio 4.84" International Journal of Engineering Research and Applications (IJERA) Vol.2, N0.7, pp 593-599, July 2012

[21]. M. Ahmed Ali Baig, S.A. Khan, and E. Rathakrishnan, Control of Nozzle Flow in Suddenly Expanded Duct with Micro Jets. International Journal of Engineering Science \& Advanced Technology [IJESAT]. 2: p. 789-795.

[22]. Ashfaq, S., S. Khan, and E. Rathakrishnan, Control of suddenly expanded flow for area ratio 3.61. International Journal of Advanced Scientific and Technical Research, 2013(3): p. 798-807. 